\title{
Efficacy and Safety of Intravenous Colistin in Very Low Birth Weight Preterm Infants
}

\author{
Ozkan Ilhan ${ }^{1}\left[\right.$ Deltem Bor ${ }^{1} \cdot$ Senem Alkan Ozdemir ${ }^{2} \cdot$ Sinem Akbay $^{3} \cdot$ Esra Arun Ozer $^{4}$
}

Published online: 3 August 2018

(c) Springer Nature Switzerland AG 2018

\begin{abstract}
Background Given the severity and high mortality of multidrug-resistant Gram-negative bacilli (MDR-GNB) infections, the use of colistin will increase in patients with MDR-GNB infection.

Objective This study aims to assess the efficacy and safety of intravenous colistin in very low birth weight (VLBW; birth weight $<1500 \mathrm{~g}$ ) preterm infants.

Methods We retrospectively analyzed the medical records of patients who received colistin between June 2016 and December 2017. The patients were assigned to two groups: the VLBW group and the non-VLBW group. Both groups were evaluated for response to treatment and adverse effects.

Results In total, 66 infants who received colistin therapy were included; of these, 28 infants were VLBW. All of our patients received standard colistin treatment of $5 \mathrm{mg} / \mathrm{kg}$ per day in three doses and the median duration of colistin treatment was 14 days. No significant differences were observed between the groups with respect to the efficacy of colistin (defined as showing microbiological clearance in control cultures and the absence of mortality during treatment) $(89.3 \mathrm{vs} 86.8 \%, p>0.99)$. Serum magnesium and potassium levels were significantly lower in the VLBW group than in the non-VLBW group during colistin therapy (magnesium, $1.30 \mathrm{vs} 1.70 \mathrm{mg} / \mathrm{dL}, p<0.001$; potassium, $3.6 \mathrm{vs} 4.6 \mathrm{mEq} / \mathrm{L}, p<0.001$ ). Acute kidney injury was observed in four infants in the VLBW group and one in the non-VLBW group, without significant differences $(p=0.15)$. Conclusions Colistin administration appears to be efficacious in VLBW infants; however, renal function tests and serum electrolytes should be monitored more closely in these infants during treatment.
\end{abstract}

Ozkan Ilhan

ozkanilhan-83@hotmail.com

1 Department of Neonatology, Harran University Faculty of Medicine, Sanliurfa, Turkey

2 Department of Neonatology, Behcet Uz Children's Hospital, Izmir, Turkey

3 Department of Neonatology, Tokat State Hospital, Tokat, Turkey

4 Department of Neonatology, Celal Bayar University Faculty of Medicine, Manisa, Turkey

\section{Key Points}

This study aimed to compare very low birth weight (VLBW) preterm infants (28 patients) and non-VLBW infants (38 patients) in terms of efficacy and safety of intravenous colistin.

While no significant differences were observed between the groups with respect to the efficacy of colistin $(89.3$ vs $86.8 \%$ ), serum magnesium and potassium levels were significantly lower in the VLBW infants than in the nonVLBW infants during colistin therapy.

Although colistin is effective in VLBW infants, renal function tests and serum electrolytes should be monitored more closely in these infants during treatment. 


\section{Introduction}

Healthcare-associated infections (HCAI) are an important cause of mortality and morbidity in neonatal intensive care units (NICUs). The incidence of HCAI has been reported to be $7-24 \%$ in NICUs $[1,2]$. In recent years, the infections caused by multidrug-resistant Gram-negative bacilli (MDR-GNB) have increased, and options in the antimicrobial agents available for treating these infections have become limited. Escherichia coli, Klebsiella pneumoniae, Pseudomonas aeruginosa, and Acinetobacter baumannii are the most common microorganisms that cause MDRGNB infections [3].

This increase in the frequency of MDR-GNB infections without new antibacterials in development has led to the consideration of the role of colistin therapy. Given the severity and high mortality of MDR-GNB infections, the use of colistin has increased in patients with MDRGNB infections [4]. In previous years, depending on the parenteral use of colistin, higher rates of adverse effects including nephrotoxicity and neurotoxicity were reported [5]. However, recent studies have shown a reduction in the rates of nephrotoxicity due to better monitoring of adverse effects in intensive care units, using less toxic colistimethate sodium, and avoiding simultaneous use of other nephrotoxic drugs [6]. Clinical data concerning the effectiveness and safety of intravenous colistin administration in neonates remain limited [6-9].

This study aimed to assess the efficacy and safety of intravenous colistin administration in the treatment of MDR-GNB infections in very low birth weight (VLBW) preterm infants compared with more mature infants.

\section{Material and Methods}

\subsection{Study Design and Data Collection}

This retrospective, single-center study was conducted at the neonatology clinic of Harran University School of Medicine (a level III neonatal intensive care unit) in Sanliurfa, Turkey, between June 2016 and December 2017. The study was approved by the local ethics committee. We included infants who were administered intravenous colistin for $>72 \mathrm{~h}$ due to MDR-GNB infections. The age of the infants included in the study ranged between 4 and 120 days. Patients with acute kidney injury (AKI) before treatment or congenital urinarytract abnormalities were excluded. Patients were assigned to one of two groups: the VLBW group (infants with birth weight $<1500 \mathrm{~g}$ ) and the non-VLBW group (infants with birth weight $>1500 \mathrm{~g}$ ).
The medical records of the participants were retrieved from hospital files. We recorded demographic data (gestational age, sex, and birth weight); underlying disease; comorbidities; surgical and invasive procedures; duration of stay in NICU; ventilator care; information regarding the administration of colistin (route, dosage, and duration) or other antibacterials (prior or concomitant to colistin therapy); use of nephrotoxic antimicrobial agents and/or medications; and laboratory tests, including microbiological (blood, urine, cerebrospinal fluid [CSF] cultures, and antibacterial sensitivity), biochemical (renal and liver functions and serum electrolytes), and hematological studies (complete blood count, C-reactive protein [CRP]). Microbiological clearance and clinical outcomes such as morbidity and mortality were also evaluated. Serum creatinine, magnesium, and potassium levels, blood urea nitrogen (BUN), and urine output were collected for assessing the possible side effects of colistin before intravenous colistin treatment, and 4-7 days after treatment was started.

\subsection{Definitions}

AKI was defined in accordance with a modified Kidney Disease: Improving Global Outcomes definition (KDIGO) staging system that Jetton and Askenazi [10] proposed on the basis of change in serum creatinin (Table 1). Standard definitions for nosocomial infections and clinical sepsis were used according to the Center for Disease Control and Prevention [11]. Severe sepsis was defined as sepsis associated with organ dysfunction, hypoperfusion, or hypotension. Multidrug resistance was considered as resistance to at least three different antibacterial groups against Gramnegative pathogens. Treatment effectiveness was evaluated based on the clinical and microbiological response. The clinical response was defined as the resolution of initial signs and symptoms, tolerance to oral feeding, and weight gain. The microbiological response was defined as the detection of no bacteria in control cultures (blood, CSF, and urine), which were taken at least 3 days after intravenous colistin treatment. The tests were repeated weekly

Table 1 Modified KDIGO definition of AKI used in the study

\begin{tabular}{ll}
\hline AKI stage & Definition \\
\hline 0 & No significant change in creatinine \\
1 & $\uparrow$ SCr by $0.3 \mathrm{mg} / \mathrm{dL}$ within $48 \mathrm{~h}$ or \\
& $\uparrow$ in SCr by $150-200 \%$ from previous trough \\
2 & $\uparrow$ in $\mathrm{SCr}$ by $200-300 \%$ from previous trough \\
3 & $\uparrow$ in $\mathrm{SCr} \geq 300 \%$ from previous trough or \\
& $\mathrm{SCr} \geq 2.5 \mathrm{mg} / \mathrm{dL}$ or \\
& $\mathrm{RRT}$ \\
\hline
\end{tabular}

$A K I$ acute kidney injury, $K D I G O$ Kidney Disease: Improving Global Outcomes, $R R T$ renal replacement therapy, $S C r$ serum creatinine 
until the treatment was completed. The incidence of the patients who showed microbiological clearance in control cultures and survived during treatment was evaluated as the outcome measure of the efficacy of colistin.

\subsection{Route of Colistin Administration and Dosage}

All infants received intravenous colistin treatment $5 \mathrm{mg} /$ $\mathrm{kg}$ per day given in three divided doses as an infusion in $5 \mathrm{~mL}$ saline over at least $30 \mathrm{~min}$. The colistin formulation comprised $150 \mathrm{mg}$ of colistin base-equivalent colistimethate sodium (Colimycin; Kocak Farma, Istanbul, Turkey). One milligram of colistimethate sodium was equal to 30,000 IU. The infants who were identified as having MDR-GNB infection based on their culture results and an outbreak of bloodstream infections caused by MDR-Klebsiella pneumoniae were administered colistin.

\subsection{Microbiologic Methods}

The BD BACTEC ${ }^{\text {TM }}$ FX40 blood culture system (BectonDickinson Diagnostics, Sparks, MD, USA) was used to isolate microorganisms from blood. Tracheal aspirates, urine, and CSF specimens were inoculated into routine culture media (5\% defibrinated sheep blood agar, chocolate agar, and an eosin-methylene blue agar plate). The microorganisms were identified by biochemical tests and the Vitek ${ }^{\circledR} 2$ Compact system (bioMérieux, Marcy l'Etoile, France). The antimicrobial susceptibility to colistin was tested with the validated disk diffusion method based on the Clinical and Laboratory Standards Institute guidelines.

\subsection{Statistical Analysis}

Data were analyzed using SPSS statistical software (version 17; Chicago, IL, USA). The Kolmogorov-Smirnov and Shapiro-Wilk tests were used to examine the distribution of data. Student's $t$ test was performed to compare continuous parametric variables, Mann-Whitney $U$ test to compare continuous nonparametric variables, and Chi-square test or Fisher's exact test to analyze categorical variables. The variables that had normal distribution were expressed as mean \pm standard deviation, nonparametric continuous variables were expressed as the median (interquartile range), and categorical variables were expressed as numbers (\%). $P$ value $<0.05$ was considered as statistically significant.

\section{Results}

In total, 1260 infants were admitted to NICU during the study period, and $152(12 \%)$ of them were diagnosed with neonatal sepsis; of these, 72 patients were administered intravenous colistin, and 66 of these patients were included. Six patients were excluded because of AKI or congenital renal anomaly before colistin therapy. Cultureproven sepsis was detected in 57 infants; the remaining nine patients received colistin empirically during an outbreak. Of the 66 patients in the study, $28(42.4 \%)$ were VLBW infants, and 38 (57.6\%) were non-VLBW infants. The primary diseases of the patients were respiratory distress syndrome (36\%); surgical diseases $(29 \%)$ such as intestinal atresia, meningomyelocele, omphalocele, gastroschisis, esophageal atresia, hydrocephalus, Hirschsprung disease; transient tachypnea of the newborn (20\%); necrotizing enterocolitis (8\%); and other causes (7\%).

Table 2 shows the demographic data, baseline characteristics, and patient outcomes. Mortality was observed in 18 of the 66 patients without significant differences between the two groups $(p=0.84)$. Favorable clinical and microbiological outcomes were observed in 58 of the 66 patients $(87.9 \%$ ) (seven died during colistin treatment, one of the deceased patients during treatment and one patient who died 30 days after the completion of colistin therapy did not show microbiological clearance); no significant differences were observed between the two groups with respect to these outcomes $(p>0.99)$. No significant differences were found for mortality due to Klebsiella spp. infection between the two groups $(p=0.35)$ (Table 2). All infants received colistin intravenously; only one patient with ventriculitis and a ventriculoperitoneal shunt received it intraventricularly. Microbiologic clearance was not observed in two patients (3\%); of these patients, one died on the eighth day of treatment, and the other one died 30 days after the completion of colistin therapy. Microorganisms were isolated from the blood cultures of $45(68 \%)$ infants, the urine cultures of $14(21 \%)$, the CSF cultures of two (3\%), and the tracheal aspirate culture of only one. The use of carbapenem as a concomitant antibiotic was statistically higher in the VLBW group than in the nonVLBW group ( $p=0.03$, Table 3$)$. In the present study, $95 \%$ of the microorganisms were resistant to penicillin, $88 \%$ to aminoglycosides, $86 \%$ to fluoroquinolones, and $85 \%$ to carbapenem; all isolated microorganisms (100\%) were sensitive to colistin and tigecycline.

The incidence of AKI was higher in the VLBW group than in the non-VLBW group (four and one patients, respectively); however, no significance difference was observed $(p=0.15)$. During colistin treatment, the serum magnesium and potassium levels were significantly lower $(p<0.001$ and $p<0.001$, respectively) and the need for additional magnesium and potassium supplementation was statistically higher $(p<0.001)$ in the VLBW group than in the non-VLBW group (Table 3 ). The serum magnesium levels were significantly lower during colistin therapy than during the start of treatment in both groups $(p<0.001)$. Regarding serum 
Table 2 Demographic characteristics and outcomes of the patients

\begin{tabular}{|c|c|c|c|}
\hline & VLBW group $(n=28)$ & Non-VLBW group $(n=38)$ & $p$ value \\
\hline Gestational age (weeks) & $28.1 \pm 1.9$ & $36.9 \pm 2.8$ & $<0.001$ \\
\hline Birth weight $(\mathrm{g})$ & $1159 \pm 248$ & $2675 \pm 607$ & $<0.001$ \\
\hline Male gender & $19(67.9 \%)$ & $24(63.2 \%)$ & 0.69 \\
\hline Cesarean section delivery & $27(96.4 \%)$ & $33(86.8 \%)$ & 0.23 \\
\hline 5th minute APGAR score & $5.1 \pm 0.6$ & $6.9 \pm 1.1$ & $<0.001$ \\
\hline Surgical disease & $4(14.3)$ & $14(36.8)$ & 0.04 \\
\hline \multicolumn{4}{|l|}{ Previous antibiotic exposure $^{a}$} \\
\hline Aminoglycoside & $1(3.6 \%)$ & $5(13.2 \%)$ & 0.23 \\
\hline Third-generation cephalosporin & $2(7.1 \%)$ & $3(7.9 \%)$ & $>0.99$ \\
\hline Carbapenem & $17(60.7 \%)$ & $22(57.9 \%)$ & 0.81 \\
\hline Vancomycin & $5(17.9 \%)$ & $12(31.6 \%)$ & 0.21 \\
\hline Amphotericin B & $2(7.1 \%)$ & $3(7.9 \%)$ & $>0.99$ \\
\hline Age at onset of infection (days) & $15(1-116)$ & $14(1-106)$ & 0.71 \\
\hline Day of colistin started (days) & $16(3-120)$ & $17(4-109)$ & 0.95 \\
\hline Duration of colistin therapy (days) & $14(3-21)$ & $14(4-21)$ & 0.28 \\
\hline Duration of hospitalization (days) & $65(14-150)$ & $41(14-174)$ & 0.13 \\
\hline \multicolumn{4}{|l|}{ Microorganisms } \\
\hline Klebsiella pneumoniae & $17(60.7 \%)$ & $24(63.2 \%)$ & 0.84 \\
\hline Escherichia coli & $3(10.7 \%)$ & $4(10.5 \%)$ & $>0.99$ \\
\hline Acinetobacter baumannii & $3(10.7 \%)$ & $3(7.9 \%)$ & 0.69 \\
\hline Sphingomonas paucimobilis & $0(0 \%)$ & $2(5.3 \%)$ & 0.50 \\
\hline Pseudomonas aeruginosa & $0(0 \%)$ & $1(2.6 \%)$ & $>0.99$ \\
\hline \multicolumn{4}{|l|}{ Outcome } \\
\hline Total mortality & $8(28.6 \%)$ & $10(27.3 \%)$ & 0.84 \\
\hline Mortality during colistin therapy & $3(10.7 \%)$ & $4(10.5 \%)$ & 0.98 \\
\hline Efficacy of colistin & $25(89.3 \%)$ & $33(86.8 \%)$ & $>0.99$ \\
\hline Mortality due to K. pneumoniae & $7(25.0 \%)$ & $6(15.8 \%)$ & 0.35 \\
\hline
\end{tabular}

Data are presented as mean \pm standard deviation, median (range) or number (\%)

$V L B W$ very low birth weight

${ }^{\mathrm{a}}$ Within 1 week before onset of infection

potassium levels during colistin treatment, although there was a significant reduction in the VLBW group $(p<0.001)$, no significant difference was observed in the non-VLBW group $(p=0.46)$ (Table 4$)$.

\section{Discussion}

In recent years, there has been an increase in the number of studies evaluating the safety and efficacy of colistin in infants, including preterm infants [6, 9, 12-15]. Favorable outcomes have ranged from 76 to $91 \%$ in infants who were administered parenteral colistin treatment $[6,9,13-17]$. Cagan et al. [6] reported that 65 neonates received colistin therapy, and microbiologic clearance was documented in all of these patients; however, 14 patients $(21 \%)$ in their study died during treatment. Jajoo et al. [13] stated that the persistence of Acinetobacter spp. on repeat culture was detected in one of the 21 patients receiving colistin therapy, and they found that the efficacy of treatment was $76 \%$. Alan et al. [9] demonstrated that colistin treatment had a high rate of efficacy, up to $80 \%$ in preterm infants with nosocomial sepsis with $A$. baumannii. In our study, the clinical efficacy was $88 \%$. This result was consistent with those from other trials in neonates. The overall mortality in neonates who have been administered colistin therapy has been reported to vary between 9 and 33\% in recent studies [6, 9, 12-16]. Jasani et al. [14] found a mortality rate of $33.7 \%$ ( 21 out of 62 ); mortality was significantly higher in neonates with lower body weights in their study. In the present study, the overall mortality was $27 \%$ (18 out of 66), which was consistent with the recent studies on newborns without significant differences between the VLBW and non-VLBW group.

The dosage of intravenous colistin recommended by manufacturers in the US is $2.5-5 \mathrm{mg} / \mathrm{kg}$ per day, divided 
Table 3 Clinical and laboratory characteristics of patients

\begin{tabular}{|c|c|c|c|}
\hline & VLBW group $(n=28)$ & Non-VLBW group $(n=38)$ & $p$ value \\
\hline Positive blood culture & $21(75.0 \%)$ & $24(63.2 \%)$ & 0.31 \\
\hline Positive urine culture & $3(10.7 \%)$ & $11(28.9 \%)$ & 0.07 \\
\hline Invasive mechanical ventilation ( $>2$ days) & $17(60.7 \%)$ & $10(26.3 \%)$ & 0.005 \\
\hline Total parenteral nutrition ( $>5$ days) & $22(78.6 \%)$ & $20(52.6 \%)$ & 0.03 \\
\hline Central venous catheter (during infection) & $19(67.9 \%)$ & $12(31.6 \%)$ & 0.004 \\
\hline CRP at onset of infection (mg/dL) & $6.4 \pm 7.4$ & $7.1 \pm 8.4$ & 0.92 \\
\hline Thrombocytopenia at onset of infection $\left(<100 \times 10^{3} / \mathrm{mm}^{3}\right)$ & $13(46.4 \%)$ & $10(26.3 \%)$ & 0.09 \\
\hline Day of microbiological clearance (days) & $4.6 \pm 1.1$ & $4.4 \pm 1.5$ & 0.63 \\
\hline Rate of microbiological clearance & $27(96.4 \%)$ & $37(97.4 \%)$ & 0.67 \\
\hline \multicolumn{4}{|l|}{ Concomitant medications } \\
\hline Diuretics (>3 doses) & $1(3.5 \%)$ & $4(10.5 \%)$ & 0.38 \\
\hline Ibuprofen & $4(14.3 \%)$ & $2(5.2 \%)$ & 0.38 \\
\hline Carbapenem & $24(85.7 \%)$ & $26(68.4 \%)$ & 0.03 \\
\hline Aminoglycoside & $2(7.1 \%)$ & $9(23.7 \%)$ & 0.07 \\
\hline Amphotericin B & $3(10.7 \%)$ & $3(7.9 \%)$ & 0.49 \\
\hline \multicolumn{4}{|l|}{ During colistin therapy } \\
\hline $\mathrm{Mg}(\mathrm{mg} / \mathrm{dL})$ (before correcting) & $1.30 \pm 0.33$ & $1.70 \pm 0.28$ & $<0.001$ \\
\hline Need for additional $\mathrm{Mg}$ supplementation & $22(78.6 \%)$ & $9(23.7 \%)$ & $<0.001$ \\
\hline $\mathrm{K}(\mathrm{mEq} / \mathrm{L})$ (before correcting) & $3.67 \pm 0.96$ & $4.64 \pm 0.72$ & $<0.001$ \\
\hline Need for additional K supplementation & $15(53.6 \%)$ & $2(5.3 \%)$ & $<0.001$ \\
\hline Calcium (mg/dL) & $8.55 \pm 1.37$ & $9.30 \pm 0.80$ & 0.02 \\
\hline Creatinine (mg/dL) & $0.54 \pm 0.26$ & $0.40 \pm 0.14$ & 0.001 \\
\hline $\mathrm{BUN}(\mathrm{mg} / \mathrm{dL})$ & $21.1 \pm 18.0$ & $13.0 \pm 13.8$ & 0.01 \\
\hline Acute kidney injury & $4(14.3 \%)$ & $1(2.6 \%)$ & 0.15 \\
\hline AST (IU/L) & $55.7 \pm 69.7$ & $39.1 \pm 36.3$ & 0.51 \\
\hline ALT (IU/L) & $27.0 \pm 35.8$ & $22.6 \pm 18.7$ & 0.13 \\
\hline
\end{tabular}

Data are presented as mean \pm standard deviation or number $(\%)$

$A L T$ alanine aminotransferase, $A S T$ aspartate transaminase, $B U N$ blood urea nitrogen, $C R P$ C-reactive protein, $K$ potassium, $M g$ magnesium, $V L B W$ very low birth weight

into 2-4 equal doses. The neonatal dosages of intravenous colistin used in recent studies ranged from $2.5 \mathrm{mg} / \mathrm{kg}$ per day to $5 \mathrm{mg} / \mathrm{kg}$ per day or from 25,000 to $75,000 \mathrm{IU} / \mathrm{kg}$ per day in 2-3 divided doses. The duration of colistin treatment ranged from 9 to 18 days $[6,12-14,18]$. In the present study, all infants were administered the standard $5 \mathrm{mg} / \mathrm{kg}$ per day in three doses, and the median duration of colistin treatment was 14 days.

Because colistin is primarily excreted by the kidneys, renal toxicity is the most common adverse effect of this drug [19]. Colistin increases the tubular epithelial cell membrane permeability, resulting in an increased influx of cations, anions, and water, leading to cell swelling and lysis [20]. This toxic mechanism may lead to electrolyte deficiency during colistin therapy. Nephrogenesis in the human fetus is not complete until approximately 34-36 weeks of gestation, with $>60 \%$ of nephrons being formed in the last trimester of pregnancy, indicating that infants born prematurely continue to form nephrons ex utero. Nephrotoxic medications may disrupt this process by decreasing the number of nephrons [21]. Conversely, premature kidneys cannot adequately handle free water, electrolytes, small proteins, bicarbonate, and drugs [22-24]. Drug treatment may significantly hamper postnatal kidney development in preterm infants by reducing the formation and number of nephrons [24].

The nephrotoxicity rates due to colistin treatment ranged from 0 to $12 \%$ in neonates $[6,9,12-16]$. Alan et al. [9] reviewed 21 preterm infants who were treated with colistin and reported a $19 \%$ incidence of nephrotoxicity. Rhone et al. [23] reviewed 107 VLBW infants who received at least one nephrotoxic medication, and the incidence of AKI was found in $27 \%$ of the patients. Patients who developed AKI had lower birth weight than those who did not develop AKI in their study. In the present study, AKI was detected in $14 \%$ of the VLBW infants (four out of 28) and in $2.6 \%$ of the control group (one out of 38); however, no significant difference was observed between the groups. 
Table 4 Change in serum electrolytes and liver function tests by colistin treatment

\begin{tabular}{lllc}
\hline & Before treatment & $\begin{array}{l}\text { During treatment } \\
\text { (4-7 days of treat- } \\
\text { ment) }\end{array}$ & $p$ value \\
\hline VLBW infants & & & \\
Mg (mg/dL) & $2.04 \pm 0.43$ & $1.30 \pm 0.33$ & $<0.001$ \\
K (mEq/L) & $4.77 \pm 0.71$ & $3.67 \pm 0.96$ & $<0.001$ \\
Calcium (mg/dL) & $8.8 \pm 1.3$ & $8.5 \pm 1.3$ & 0.54 \\
AST (IU/L) & $33 \pm 19$ & $55 \pm 69$ & 0.08 \\
ALT (IU/L) & $13 \pm 6$ & $27 \pm 35$ & 0.04 \\
Non-VLBW group & & & $<0.001$ \\
Mg (mg/dL) & $1.96 \pm 0.17$ & $1.70 \pm 0.28$ & 0.46 \\
K (mEq/L) & $4.63 \pm 0.71$ & $4.64 \pm 0.72$ & 0.27 \\
Calcium (mg/dL) & $9.1 \pm 0.6$ & $9.3 \pm 0.8$ & 0.11 \\
AST (IU/L) & $30 \pm 16$ & $39 \pm 36$ & 0.06 \\
ALT (IU/L) & $17 \pm 10$ & $22 \pm 18$ &
\end{tabular}

Data are presented as mean \pm standard deviation or number (\%)

$A L T$ alanine aminotransferase, $A S T$ aspartate transaminase, $K$ potassium, $M g$ magnesium, $V L B W$ very low birth weight

Clinical improvement was achieved in two of the five patients after discontinuation of colistin without requiring renal replacement therapy. Similar to the studies conducted by Rhone et al. [23] and Alan et al. [9], we found a higher incidence of AKI in infants with lower birth weight, without a significant difference. Consistent with the report by Alan et al. [9], 53\% of our infants required potassium supplementation; however, in the present study, the need for additional magnesium supplementation was higher than that in the Alan study (78 vs 52\%, respectively). Lower serum magnesium and potassium levels in VLBW infants during treatment may be related to a decreased number of nephrons in preterm infants. An adult study by Pogue et al. [25] reported that patients who developed nephrotoxicity received significantly higher colistin doses than those who did not (5.48 vs $3.95 \mathrm{mg} / \mathrm{kg}$ per day). The cause of the high rates of electrolyte imbalance in our study, particularly in VLBW infants, may be associated with all of our patients receiving standard colistin treatment of $5 \mathrm{mg} / \mathrm{kg}$ per day.

Neurotoxicity is another adverse effect of intravenous colistin therapy. Orofacial paresthesias, visual disturbances, vertigo, mental confusion, ataxia, and seizure are neurological side effects of colistin use; however, only apnea and convulsions are recognizable neurological findings in neonates $[9,26]$. It is difficult to detect neurological symptoms in preterm infants. As the previous clinical conditions may lead to similar symptoms, it may be difficult to distinguish the neurological side effects of colistin therapy [9]. Most of our patients were premature or receiving sedation/analgesia; therefore, we considered that adverse neurological events, such as apnea and convulsions, could not be attributed to the colistin treatment in the present study.

There are some limitations to our study. First, the present study was a retrospective study. Second, it was difficult to assess the neurotoxic and nephrotoxic adverse effects because of confounding factors such as administration of sedation to the patients, prematurity, concomitant nephrotoxic medications, sepsis, and shock. Finally, given the concomitant use of other drugs, particularly antibacterials in addition to colistin, adverse effects cannot be attributed solely to colistin.

\section{Conclusion}

Colistin administration appears to be efficacious in VLBW infants with MDR infection; however, renal function tests and serum electrolytes should be monitored more closely in these infants during treatment. Additional pharmacokinetic and pharmacodynamic studies are needed to determine the optimal dose of colistin in newborn infants with lower birth weight.

\section{Compliance with Ethical Standards}

Funding This study did not receive any specific funding.

Conflict of interest Ozkan Ilhan, Meltem Bor, Senem Alkan Ozdemir, Sinem Akbay and Esra Arun Ozer have no conflicts of interest to declare.

\section{References}

1. Aly H, Herson V, Duncan A, Herr J, Bender J, Patel K, et al. Is bloodstream infection preventable among premature infants? A tale of two cities. Pediatrics. 2005;115:1513-8.

2. Stoll BJ, Hansen N, Fanaroff AA, Wright LL, Carlo WA, Ehrenkranz RA, et al. Late-onset sepsis in very low birth weight neonates: the experience of the NICHD Neonatal Research Network. Pediatrics. 2002;110:285-91.

3. Karaiskos I, Galani L, Baziaka F, Giamarellou H. Intraventricular and intrathecal colistin as the last therapeutic resort for the treatment of multidrug-resistant and extensively drug-resistant Acinetobacter baumannii ventriculitis and meningitis: a litera-ture review. Int J Antimicrob Agents. 2013;41:499-508.

4. Lee HY, Chiu CH. Efficacy and safety of using colistin in neonates. Pediatr Neonatol. 2017;58:473-4.

5. Koch-Weser J, Sidel VW, Federman EB, Kanarek P, Finer DC, Eaton AE. Adverse effects of sodium colistimethate. Manifestations and specific reaction rates during 317 courses of therapy. Ann Intern Med. 1970;72:857-68.

6. Cagan E, Kıray Bas E, Asker HS. Use of colistin in a neonatal intensive care unit: a cohort study of 65 patients. Med Sci Monit. 2017;23:548-54. 
7. Falagas ME, Vouloumanou EK, Rafailidis PI. Systemic colistin use in children without cystic fibrosis: a systematic review of the literature. Int J Antimicrob Agents. 2009;33(503):e1-13.

8. Iosifidis E, Antachopoulos C, Ioannidou M, Mitroudi M, Sdougka M, Drossou-Agakidou V, et al. Colistin administration to pediatric and neonatal patients. Eur J Pediatr. 2010;169:867-74.

9. Alan S, Yildiz D, Erdeve O, Cakir U, Kahvecioglu D, Okulu E, et al. Efficacy and safety of intravenous colistin in preterm infants with nosocomial sepsis caused by Acinetobacter baumannii. Am J Perinatol. 2014;31:1079-86.

10. Jetton JG, Askenazi DJ. Update on acute kidney injury in the neonate. Curr Opin Pediatr. 2012;24:191-6.

11. Horan TC, Andrus M, Dudeck MA. CDC/NHSN surveillance definition of health care-associated infection and criteria for specific types of infections in the acute care setting. Am J Infect Control. 2008;36:309-32.

12. İpek MS, Aktar F, Okur N, Celik M, Ozbek E. Colistin use in critically ill neonates: a case-control study. Pediatr Neonatol. 2017;58:490-6.

13. Jajoo M, Kumar V, Jain M, Kumari S, Manchanda V. Intravenous colistin administration in neonates. Pediatr Infect Dis J. 2011;30:218-21.

14. Jasani B, Kannan S, Nanavati R, Gogtay NJ, Thatte U. An audit of colistinuse in neonatal sepsis from a tertiary care centre of a resource-limited country. Indian J Med Res. 2016;144:433-9.

15. Nag SS, Dutta A, Mitra P, Majumdar R, Chatterjee M. Intravenous colistimethate sodium in neonatal sepsis. Indian J Pediatr. 2016;83:864-5.

16. Al-Lawama M, Aljbour H, Tanash A, Badran E. Efficacy and safety of intravenous colistin in preterm infants with nosocomial sepsis caused by Acinetobacter baumannii. Ann Clin Microbiol Antimicrob. 2016;15:8

17. Serafettin Tekgunduz K, Kara M, Caner I, Demirelli Y. Safety and efficacy of intravenous colistin in neonates with culture proven sepsis. Iran J Pediatr. 2015;25:e453.

18. Choudhry S, Ahmad E, Batool A, Raja N. Use of colistin for the treatment of multi drug resistant isolates in neonates. J Pak Med Assoc. 2017;67:1157-60.

19. Lewis JR, Lewis SA. Colistin interactions with the mammalian urothelium. Am J Physiol Cell Physiol. 2004;286:913-22.

20. Berg JR, Spilker CM, Lewis SA. Effects of polymyxin B on mammalian urinary bladder. J Membr Biol. 1996;154(2):119-30.

21. Mackenzie HS, Brenner BM. Fewer nephrons at birth: a missing link in the etiology of essential hypertension? Am J Kidney Dis. 1995;26:91-8.

22. Stritzke A, Thomas S, Amin H, Fusch C, Lodha A. Renal consequences of preterm birth. Mol Cell Pediatr. 2017;4:2.

23. Rhone ET, Carmody JB, Swanson JR, Charlton JR. Nephrotoxic medication exposure in very low birth weight infants. J Matern Fetal Neonatal Med. 2014;27:1485-90.

24. Schreuder MF, Bueters RR, Allegaert K. The interplay between drugs and the kidney in premature neonates. Pediatr Nephrol. 2014;29:2083-91.

25. Pogue JM, Lee J, Marchaim D, Yee V, Zhao JJ, Chopra T, et al. Incidence of and risk factors for colistin associated nephrotoxicity in a large academic health system. Clin Infect Dis. 2011;53:879-84.

26. Spapen H, Jacobs R, Van Gorp V, Troubleyn J, Honoré PM. Renal and neurological side effects of colistin in critically ill patients. Ann Intensive Care. 2011;1:14. 